\title{
Train-induced pressure on pedestrians
}

\author{
A. Sanz-Andrés*, J. Santiago-Prowald
}

\begin{abstract}
A simple analytical model for the train-induced flow and its effects on pedestrians is presented in this paper. The expressions developed for the induced air velocity and pressure on the pedestrian surface, as well as their dependence with time, are obtained from unsteady potential theory. The relevant parameters and their effects are analysed, in particular the sensitivity of the pressure coefficient and its rate of change on the train and pedestrian transverse size, the distance to the tracks and the pressure measurement location on the pedestrian surface. In spite of the extreme simplicity of the model and the expressions obtained, good correlation is observed with previously existing experiments. With this work, an absence of published studies concerning analytical approaches to the problem of vehicleinduced pressure on pedestrians is intended to be covered, allowing for simplified testing procedures.
\end{abstract}

\section{Introduction}

High-speed trains are becoming a popular means of transportation for offering a convenient connection between business centres and even touristic locations. However, there is still an open issue concerning passenger safety and comfort inside train stations. In particular, the air flows induced by passing trains on pedestrians waiting on platforms can be as severe as those occurring in storms, and therefore dangerous when not unpleasant. 
The relevant factors governing how people feel the effects of wind have originated detailed studies for decades [1-3]. Most of the criteria developed for pedestrian comfort are based on probabilistic procedures that are not directly applicable to vehicle-induced flow. However, it is widely accepted that pressure rates of change and gradients such as those induced by gusts, play a relevant role in pedestrian safety and comfort $[4,5]$.

Concerning train-generated air flows, there are a significant amount of studies on train motion in tunnels, the compression wave generated in the tunnel, the booming noise when the compression wave reaches the end of the tunnel, and the effect on the aerodynamic drag of the train [6-8], but there is a lack of systematic studies concerning the effect on the pedestrian standing on the train station platform.

In this paper, a simple model to explain this effect is presented. It provides a grasp of the physical concepts playing a role in the process as well as the parameters involved. The solution presented for simplified train and pedestrian models is based on unsteady incompressible potential flow theory. This solution has been compared to experimental data [9]. In spite of the simplicity of the model and the solution obtained, as well as the uncertainty of the parameters, surprisingly good correlation has been obtained, bearing good expectations for the future development of tests.

\section{Problem definition}

As a train passes through a train station, it generates air motion that can be unpleasant and even dangerous to the pedestrians.

The train speed in this situation can reach $150 \mathrm{~km} / \mathrm{h}$, which is low enough to consider the fluid motion as incompressible. The fluid motion as seen by an observer placed in the train can be considered as steady, but for an observer placed at the station the fluid flow is unsteady and can be regarded as a gust. This unsteady flow can generate large loads on the obstacles (e.g. pedestrians) placed on the platforms.

In a first step, the flow field in the train reference frame is described by using a potential axisymmetric model when the train is moving at constant speed, $U_{\infty}$. Afterwards, the unsteady flow field seen by an observer placed at the train station is obtained by a coordinate transformation.

Then, the stagnation pressure on a point placed on a body (representative of the pedestrian) is obtained.

Let us consider the surface of the train body as the upper half of an axisymmetric body which is obtained in a potential incompressible flow by superposition of a uniform, steady, incident flow with speed $U_{\infty}$ and a constant flow rate source, $Q$, as shown in Fig. 1.

The station platform is represented by the $(x, y)$ plane. The source flow rate, $Q$, is obtained by using the mass conservation at great distance downstream of the source where the flow speed is close to $U_{\infty}$ :

$$
Q=\pi r_{\mathrm{t}}^{2} U_{\infty}
$$

where $r_{\mathrm{t}}$ is the radius of the train's circular cross-section. 


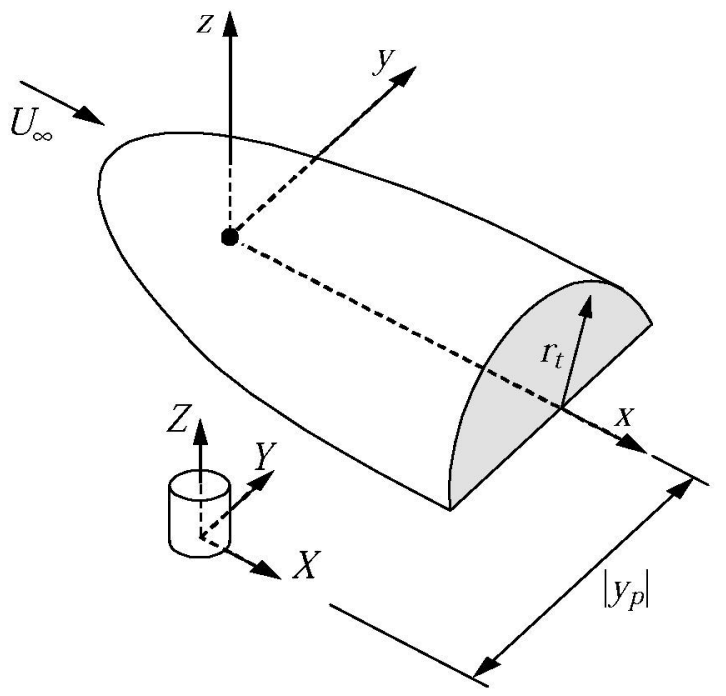

Fig. 1. Modelization of the flow around a train moving at speed $U_{\infty} .(x, y, z)$ train fixed reference system. $(X, Y, Z)$ station fixed reference frame. $r_{\mathrm{t}}$ : radius of the train cross-section. $y_{\mathrm{p}}$ : distance of train path to pedestrian location.

The velocity field is axisymmetric around the $x$-axis. Let us assume that there is a velocity potential $\varphi$ from which the speed components can be derived:

$$
u=\varphi_{x}, \quad w=\varphi_{r},
$$

where $u$ and $w$ are the longitudinal and radial components, respectively, and the radial coordinate $r=\sqrt{y^{2}+z^{2}}$. The velocity field, according to the above assumptions, is given by the following expressions:

$$
\begin{aligned}
& u=U_{\infty}\left[1+\frac{r_{\mathrm{t}}^{2}}{4} \frac{x}{\left(r^{2}+x^{2}\right)^{3 / 2}}\right], \\
& w=U_{\infty} \frac{r_{\mathrm{t}}^{2}}{4} \frac{r}{\left(r^{2}+x^{2}\right)^{3 / 2}} .
\end{aligned}
$$

This velocity field, in a reference frame that is fixed to the station platform (axes $X, Y, Z)$, denoted as $(U, W)$, is a non-steady one, and it is obtained by using the following reference frame transformation (remember that the origin of reference system $x, y, z$ moves with speed $-U_{\infty} i$ with regard to the axes $X, Y, Z$ ):

$$
\begin{aligned}
& X=x-U_{\infty} t, \\
& Y=y+y_{\mathrm{p}}, \\
& Z=z,
\end{aligned}
$$


which leads to the following expressions:

$$
\begin{aligned}
& U=u-U_{\infty}=U_{\infty} \frac{r_{\mathrm{t}}^{2}}{4} \frac{X+U_{\infty} t}{\left[r^{2}+\left(X+U_{\infty} t\right)^{2}\right]^{3 / 2}}, \\
& W=w=U_{\infty} \frac{r_{\mathrm{t}}^{2}}{4} \frac{r}{\left[r^{2}+\left(X+U_{\infty} t\right)^{2}\right]^{3 / 2}} .
\end{aligned}
$$

This velocity field is the same as a fixed observer can feel when in presence of a source moving at $-U_{\infty} \boldsymbol{i}$ speed in an incompressible fluid.

The vector $(U, W)$ modulus is

$$
V_{\mathrm{p}}=\sqrt{U^{2}+W^{2}}=U_{\infty} \frac{r_{\mathrm{t}}^{2}}{4} \frac{1}{r^{2}+\left(X+U_{\infty} t\right)^{2}} .
$$

This is the speed seen by a pedestrian standing still on the platform at a point $(X, Y)$.

Let us represent this pedestrian as a cylinder of circular cross-section, with radius $r_{\mathrm{p}}$, under an impinging speed $V_{\mathrm{p}}(t)$, as shown in Fig. 2. As $r_{\mathrm{p}}$ is much smaller than the cylinder length (the pedestrian height), we assume the velocity field around the cylinder as two-dimensional. The pedestrian reference system is defined as shown in the figure. The $X^{\prime}$-axis is aligned with the train path and the impinging speed forms an angle $\alpha$ with it.

Let us assume that the flow field around the cylinder is derived from an unsteady potential function $\phi\left(X^{\prime}, Y^{\prime}, t\right)$. As $V_{\mathrm{p}}=V_{\mathrm{p}}(t)$ is a function of time, the pressure distribution on the surface can be obtained by using the Bernoulli equation for potential unsteady incompressible flow:

$$
\phi_{t}+\frac{1}{2}|\nabla \phi|^{2}+\frac{p}{\rho}=C(t)
$$

where the right-hand side is a time dependent uniform term that is trivially obtained from a proper treatment of the boundary conditions. From Eq. (5) the general

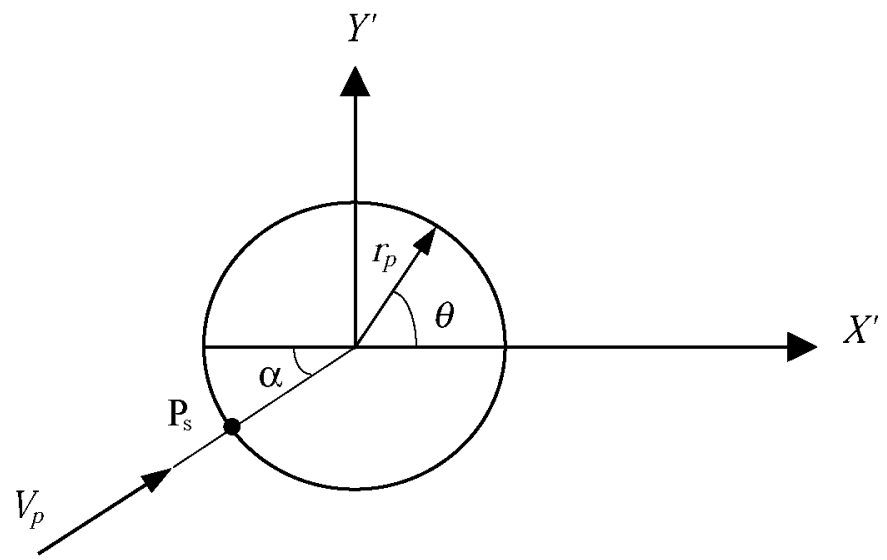

Fig. 2. Model of the pedestrian: uniform flow $V_{\mathrm{p}}$ impinging on a circular cylinder of radius $r_{\mathrm{p}}$. 
expression for the pressure coefficient on the cylinder, referred to the train speed $U_{\infty}$, is given by

$$
c_{p}=\frac{p-p_{\infty}}{\frac{1}{2} \rho U_{\infty}^{2}}=\left(\frac{V_{\mathrm{p}}}{U_{\infty}}\right)^{2}-\frac{2 \phi_{\mathrm{t}}+|\nabla \phi|^{2}}{U_{\infty}^{2}} .
$$

The velocity potential on the cylinder, $\phi$, in which time is treated as a parameter coming from the expressions of both $V_{\mathrm{p}}$ and the incidence angle $\alpha$, is given by

$$
\phi(\theta, t)=2 r_{\mathrm{p}} V_{\mathrm{p}}(t) \cos (\theta-\alpha(t)) \text {. }
$$

In the particular case of the stagnation point $\mathrm{P}_{\mathrm{S}}\left(\theta_{\mathbf{P}_{\mathrm{S}}}=\pi+\alpha(t)\right)$, the fluid velocity is zero and therefore:

$$
c_{p}\left(\mathrm{P}_{\mathrm{S}}\right)=\left(\frac{V_{\mathrm{p}}}{U_{\infty}}\right)^{2}-\frac{2 \phi_{\mathrm{t}}}{U_{\infty}^{2}},
$$

such that

$$
\phi_{t}\left(\mathrm{P}_{\mathrm{S}}\right)=-2 r_{\mathrm{p}} \frac{\mathrm{d} V_{\mathrm{p}}}{\mathrm{d} t}
$$

Here the air speed $V_{\mathrm{p}}=V_{\mathrm{p}}(t)$ should be evaluated at the location of the pedestrian, assuming that the effect of the cylinder on the impinging flow is negligible, except when very close to the cylinder. Then, from Eq. (4) with $X=0$ and $r=y_{\mathrm{p}}$, we obtain

$$
V_{\mathrm{p}}=U_{\infty} \frac{r_{\mathrm{t}}^{2}}{4} \frac{1}{y_{\mathrm{p}}^{2}+U_{\infty}^{2} t^{2}} .
$$

The pressure coefficient $c_{p}$ at the stagnation point is then

$$
c_{p}\left(\mathrm{P}_{\mathrm{S}}\right)=\frac{R_{\mathrm{t}}^{4} / 16-2 R_{\mathrm{p}} R_{\mathrm{t}}^{2} T}{\left(1+T^{2}\right)^{2}},
$$

where $T=t U_{\infty} / y_{\mathrm{p}}$ is the dimensionless time, $R_{\mathrm{p}}=r_{\mathrm{p}} / y_{\mathrm{p}}$ and $R_{\mathrm{t}}=r_{\mathrm{t}} / y_{\mathrm{p}}$ are the dimensionless geometrical parameters of the problem. The distance $y_{\mathrm{p}}$ is used as characteristic length. Note that the relevant cause of the pressure variation is the rate of change of the incident speed, $V_{\mathrm{p}}$.

The variation with time, $T$, of the pressure coefficient, $c_{p}$, at the stagnation point is shown in Fig. 3, in which $T<0$ represents the time interval before the passing of the moving reference frame origin (where the source is placed) across the plane $X=0$ (where the pedestrian is placed).

\section{Comparison with experiments}

The availability of experimental data about pressure induced on pedestrians by moving objects is very limited in the literature. The results in [9] include pressure measurements on pedestrians induced by high-speed trains passing along station platforms. These tests have been performed on a 1:50 scale train model moved impulsively by means of a pneumatic cylinder and measuring pressure as a function 


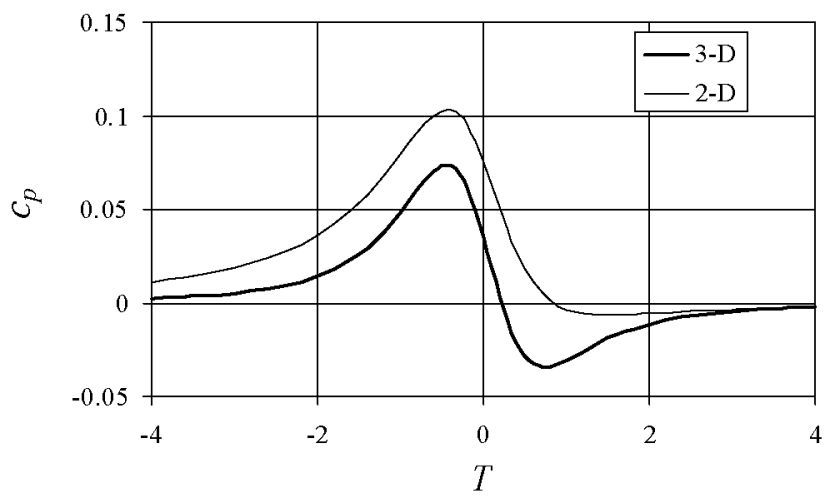

Fig. 3. Variation with the dimensionless time, $T$, of the pressure coefficient, $c_{p}$, at the stagnation point on the cylinder representing the pedestrian body. $T=0$ is the time instant when the origin of the moving axis passes at the passenger position ( $X=0$ ). Results obtained for $r_{\mathrm{t}}=1.46 \mathrm{~m}, y_{\mathrm{p}}=1.69 \mathrm{~m}, r_{\mathrm{p}}=0.18 \mathrm{~m}$. The solid line results from axisymmetric train shape and dotted line results from a $2-\mathrm{D}$ train shape (as an approximation for a tall train under a station roof).

of time on a plate resembling the pedestrian. However, very scarce information about test set-up, conditions and results is presented in that paper.

Experiments measuring the loads induced on an overhead highway sign structure by vehicle-induced gusts are presented in [10]. No attempt has been made to compare quantitatively these results with the model because the parameters of this experimental configuration are very far from the assumptions made in the derivation of the model (the size of the sign is not small compared with the size of the truck that induces the load, the truck has a very bluff nose and the length is very short, making difficult its representation by just one or two sources, etc.). However, qualitatively the curves of the time variation of the pressure have a shape that is similar to the one shown in Fig. 3 (up to the point where data are comparable, i.e., excluding structural response and base-flow phenomena).

In this section the theoretical model developed above is used to correlate the mentioned test data in [9]. However, it is difficult to derive all the parameters required from the information supplied in [9]. For this reason, some assumptions and approximate data fitting have been carried out, explained below, and hence the following parameter values are used hereafter in order to compare with the experimental data:

$$
r_{\mathrm{p}}=0.18 \mathrm{~m}, \quad r_{\mathrm{t}}=1.46 \mathrm{~m}, \quad r_{\mathrm{b}}=1.69 \mathrm{~m} .
$$

These values should be understood as the result of adjusting the theoretical model in order to qualitatively capture the behaviour of the measured data. An accurate correlation is not intended, although surprisingly good results are obtained. The relevant finding is the reproduction of the initial pressure rise followed by a suction peak with a steep drop in between, which are the main characteristics of the load 
exerted on the pedestrian. Obviously, a detailed model of the train and pedestrian shapes would give better results, especially for the pressure peak values.

Furthermore, the dimensionless time employed in the above-mentioned experiments is

$$
\tau=\frac{t U_{\infty}}{\lambda}
$$

where $\lambda$ is referred in [9] as "the influence length of the pressure wave". A value of $\lambda=0.2 \mathrm{~m}$ is given for the full-scale problem. In order to compare the full-scale analytical model and the 1:50 test-scale data, the following time-scale transformation has been used:

$$
T=\frac{U_{\infty}}{y_{\mathrm{p}}} \frac{\lambda^{\mathrm{t}}}{U_{\infty}^{\mathrm{t}}} \tau,
$$

where the superscript $\mathrm{t}$ stands for test data, $U_{\infty}^{\mathrm{t}}=14 \mathrm{~m} / \mathrm{s}$ and $\lambda^{\mathrm{t}}=0.2 / 50 \mathrm{~m}$. The correlation result is shown in Fig. 4, in which the pressure coefficient at three fixed points on the circular cylinder, together with the stagnation point, compare rather well with the experimental data, except for the initial pressure rise.

It has to be mentioned that the pressure measurements have been obtained at a single location on a plate of unknown size, representing the pedestrian, placed at an unknown distance to the train path. For this reason, the parameter values given above result from a fitting process of non-dimensional time scales and pressure coefficient rate of change. For the stagnation point this pressure rate of change,
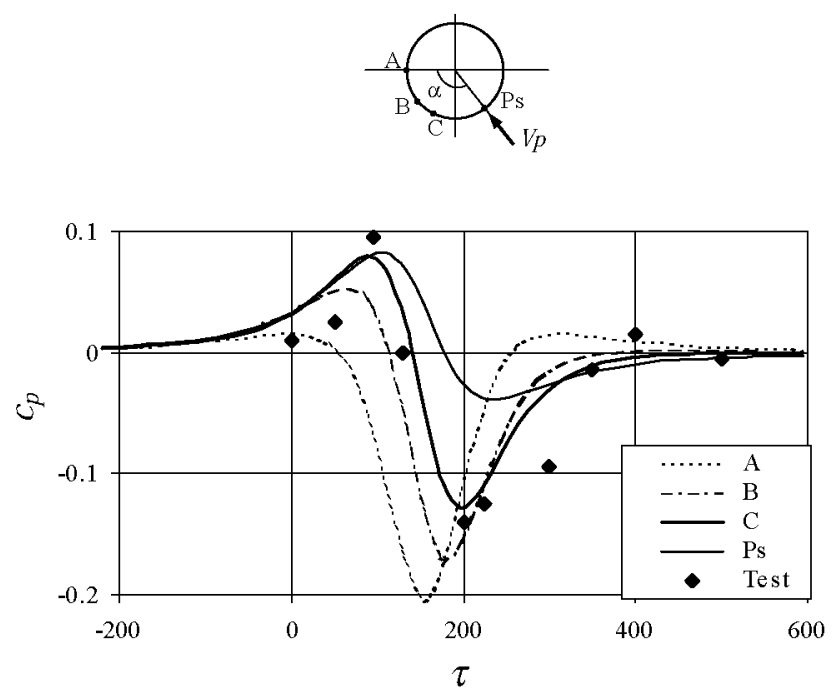

Fig. 4. Evolution of pressure coefficient, $c_{p}$, with the non-dimensional time $\tau$ at four different locations on the cylinder: $\mathrm{A}, \mathrm{B}$ and $\mathrm{C}$ obtained from Eq. (6) and the stagnation point $\left(\mathrm{P}_{\mathrm{S}}\right.$ ), obtained from Eq. (10). Parameter values: $r_{\mathrm{t}}=1.46 \mathrm{~m}, y_{\mathrm{p}}=1.69 \mathrm{~m}, r_{\mathrm{p}}=0.18 \mathrm{~m}$. Comparison with experimental results [9]. 
which is a good estimate of the load on the passenger, obeys a very simple relation:

$$
\left.\frac{\mathrm{d} c_{p}}{\mathrm{~d} T}\right|_{T=0}=-2 \frac{r_{\mathrm{p}} r_{\mathrm{t}}^{2}}{y_{\mathrm{p}}^{3}}=-2 R_{\mathrm{p}} R_{\mathrm{t}}^{2}
$$

or in terms of the experimental time-scale $\tau$ :

$$
\left.\frac{\mathrm{d} c_{p}}{\mathrm{~d} \tau}\right|_{\tau=0}=-2 \frac{U_{\infty} \lambda^{\mathrm{t}}}{U_{\infty}^{\mathrm{t}} y_{\mathrm{p}}} \frac{r_{\mathrm{p}} r_{\mathrm{t}}^{2}}{y_{\mathrm{p}}^{3}},
$$

where the first quotient comes from the time-scale transformation. In Fig. 4 the initial time for analytical curves has been offset to capture the instant of train passage with the test data for a certain location on the cylinder. Using the assumed parameters we obtain at the stagnation point during train passage $\mathrm{d} c_{p} / \mathrm{d} \tau=$ $-1.4 \times 10^{-3}$ by means of Eq. (14), while $-1.8 \times 10^{-3}$ is reported in [9]. This pressure rate of change, however, is much better obtained at locations other than the stagnation point, as for example point $\mathrm{C}$ in Fig. 4 , for which $\mathrm{d} c_{p} / \mathrm{d} \tau$ is the same as for the test data.

The high variability of pressure response is a consequence of the sensitivity of pressure output upon the measurement location and even the shape of the cylinder cross-section. Therefore, future experiments should take this consideration into account.

It should be noted in (13) that the pressure rate decreases quickly with the distance from the pedestrian to the train axis as $y_{\mathrm{p}}^{-3}$, and increases with the train cross-section area $\left(r_{\mathrm{t}}^{2} \pi / 2\right)$ and with the pedestrian size $\left(r_{\mathrm{p}}\right)$.

\section{Conclusions}

The model presented in this paper is just a simple estimation exercise in order to show the relevant parameters and physical phenomena involved in the assessment of pressure loads on pedestrians induced by trains or similar vehicles. In this way, the design of future experiments can be improved.

The analytical expressions developed are based on unsteady potential theory, modelling the train by a 2-D or 3-D source moving along a straight path, and the pedestrian by a circular cylinder on which the train-induced flow impinges with an angle varying with time.

Very limited information has been found in the literature. Moreover, the available test data are provided without the necessary details for performing a proper correlation. Therefore, some of the parameters have been assessed and others have been deduced from approximate fitting. However, in spite of the simplicity of the model and the uncertainty of the parameters, surprisingly good correlation has been obtained. This concerns pressure levels and their evolution with time, except for the initial pressure rise, which may be affected by the particular design of the train head. In particular, good agreement between experiments and model is achieved for the dependency of the pressure rate of change on the distance to the train path, $y_{\mathrm{p}}$, which is observed to behave as $y_{\mathrm{p}}^{-3}$. 
One of the results obtained from the model is the sensitivity of the pressure load upon the measurement location on the pedestrian surface. This geometrical parameter, as well as the passenger cross-section, should be considered for future tests, not only the distance to the tracks or the train size. Furthermore, using this model it may also be possible to simplify the test procedure by keeping the train fixed in a wind tunnel and performing velocity measurements at different locations along a parallel line. Afterwards, the unsteady load on the real-scale passenger can be estimated by the reference frame and time-scale transformations given in this paper.

\section{Acknowledgements}

The authors wish to thank J. Meseguer, J.M. Perales and G. Tévar for fruitful discussions.

\section{References}

[1] W.H. Melbourne, Criteria for environmental wind conditions, J. Ind. Aerodyn. 3 (1978) 241-249.

[2] F.H. Durgin, Pedestrian level wind criteria using the equivalent average, J. Wind Eng. Ind. Aerodyn. 66 (1997) 215-226.

[3] M.J. Soligo, P.A. Irwin, C.J. Williams, G.D. Schuyler, A comprehensive assessment of pedestrian comfort including thermal effects, J. Wind Eng. Ind. Aerodyn. 77-78 (1998) 753-766.

[4] J.C.R. Hunt, E.C. Poulton, J.C. Mumford, The effects of wind on people: new criteria based on wind tunnel experiments, Build. Environ. 11 (1976) 15-28.

[5] S. Murakami, K. Uehara, K. Deguchi, Wind effects on pedestrians: new criteria based on outdoor observations of over 2000 persons, in: J.E. Cermak (Ed.), Wind Engineering, Proceedings of the Fifth International Conference, Fort Collins, CO, USA, Pergamon Press, Oxford, 1979, pp. 277-288.

[6] M.S. Howe, Mach number dependence of the compression wave generated by a high-speed train entering a tunnel, J. Sound Vib. 212 (1998) 23-36.

[7] T. Ogawa, K. Fujii, Numerical investigation of three-dimensional compressible flows induced by a train moving into a tunnel, Comput. Fluids 26 (1997) 565-585.

[8] M. Sajben, Fluid mechanics of train-tunnel systems in unsteady motion, AIAA J. 9 (1971) 1538-1545.

[9] H.J. Gerhardt, O. Krüger, Wind and train driven air movements in train stations, J. Wind Eng. Ind. Aerodyn. 74-76 (1998) 589-597.

[10] P.M. Cali, E.E. Covert, Experimental measurements of loads on an overhead highway sign structure by vehicle-induced gusts, J. Wind Eng. Ind. Aerodyn. 84 (2000) 87-100. 